\title{
Cardiovascular and autonomic response induced by a 20-week military training programme in young healthy South African males
}

\author{
P C Zondi, ${ }^{1}$ MB ChB, MSc (Sports Med), MBA; D C Janse van Rensburg, ${ }^{1}$ MD; C C Grant, ${ }^{1} \mathrm{PhD}$ (Physiology); L Fletcher, ${ }^{2} \mathrm{PhD}($ Statistics)
}

${ }^{1}$ Section Sports Medicine, Department of Orthopaedics, Faculty of Health Sciences, University of Pretoria, South Africa

${ }^{2}$ Department of Statistics, University of Pretoria, South Africa

Corresponding author: P C Zondi (phatho.cele@up.ac.za)

\begin{abstract}
Background. Studies investigating the relationship between exercise and haemodynamic regulation conducted in older caucasian and black African populations suggest that lifestyle modification is effective in the management of numerous disease processes. There are few longterm studies in young healthy populations and even less is known about the influence of habitual exercise on autonomic and haemodynamic variables in young black African subjects.

Objective. To investigate the benefit of prolonged exercise on cardiovascular and haemodynamic variables in young healthy black African males.

Methods. Fifty-five healthy male volunteers between 18 and 22 years of age participated in this prospective 20-week medium- to highintensity exercise intervention study with a self-control design. The Finometer Pro (Finapres Medical Systems, the Netherlands) was used for non-invasive data sampling of a number of cardiovascular and autonomic variables. Results were generated by computer algorithm and were analysed using non-parametric Wilcoxon signed rank tests.

Results. Significant $(p<0.05)$ cardiovascular changes included an increase in aerobic capacity, stroke volume, cardiac output and ejection fraction, and a decrease in diastolic blood pressure and heart rate. There was a significant decrease in total peripheral resistance and ascending aorta impedance. Systolic blood pressure, mean arterial pressure and arterial compliance remained unchanged, while the decrease in blood pressure variability was not statistically significant $(p>0.05)$.

Conclusion. The measured changes suggest a favourable response to exercise and imply that habitual exercise may be an important lifestyle modification for reducing the risk of cardiovascular disease in young black African males.
\end{abstract}

S Afr J Sports Med 2015;27(2):28-32. DOI:10.7196/SAJSM.564

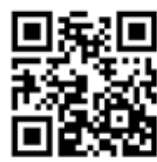

Epidemiological studies show that, compared with their caucasian peers, individuals of black African descent have a higher prevalence of cardiac risk factors. ${ }^{[1,2]}$ Black Africans have stiffer large central arteries, impaired dilation of small peripheral arteries and a greater prevalence of hypertension, diabetes and cardiovascular disease..$^{[2-4]}$

It is well established that a number of cardiovascular indices, such as arterial stiffness, blood pressure and autonomic regulation, are differentially affected by ageing. ${ }^{[5]}$ The influence of western lifestyle (sedentary behaviour and increased consumption of high-energy processed foods) and rural urbanisation in sub-Saharan Africa has seen a shift in the underlying population distribution of risk factors for cardiovascular disease. ${ }^{[6]}$ Younger populations are increasingly affected by metabolic and cardiovascular illness; in America, the percentage of youths with chronic disease has almost quadrupled in the past 45 years, with racial minority children being disproportionately affected. ${ }^{[7]}$ Research shows that an increase in childhood chronic diseases results in an increase in adulthood illnesses, ${ }^{[7]}$ and as such, methods need to be sought to curb this increasing epidemic.

Chronic inactivity is associated with increased cardiovascular risk and is a key factor in the progression of chronic disease. ${ }^{[5,8]}$ Conversely, regular physical activity has been shown to result in structural and functional vascular adaptations that protect against cardiovascular and metabolic disease. ${ }^{[5,8]}$ The majority of studies examining the effect of exercise on cardiac and vascular function have been conducted on caucasian subjects and have found that major adaptations include a lowered resting heart rate, increased stroke volume, improved limb blood flow and limb vasodilatory capacity, and a decrease in arterial stiffness. ${ }^{[9]}$ Autonomic adaptations include increased vagal tone and baroreflex sensitivity ${ }^{[9,11]}$ Several studies that have been conducted in older African populations suggest that regular exercise has beneficial cardiovascular effects and may decrease the risk for cardiac complications. ${ }^{[10]}$ There are fewer studies conducted among young populations in general, ${ }^{[2]}$ and little is known about the cardiovascular and autonomic effects of habitual exercise in young healthy African subjects. Knowing that black Africans are at an increased risk of cardiovascular disease, and that younger populations in general are increasingly being affected by metabolic disease, it becomes important to establish if physical activity infers similarly protective adaptations in young black subjects as it does in older black and caucasian populations.

As such, the purpose of this study was to investigate the effect of habitual physical activity in young healthy black African males by documenting changes in autonomic and haemodynamic markers after a 20-week exercise intervention. Markers monitored were those tested in previous studies investigating the effect of exercise on cardiac and autonomic function and included: aerobic capacity $\left(\mathrm{VO}_{2}\right.$ max), systolic blood pressure (SBP), diastolic blood pressure (DBP), 
mean arterial blood pressure (MAP), blood pressure varibilty (BPV), cardiac output (CO), stroke volume (SV), heart rate (HR), total peripheral resistance (TPR), ejection fraction (EJT), ascending aorta impedance $(\mathrm{ZaO})$ and arterial compliance (Cwk). It was hypothesised that 20 weeks of graduated exercise training would result in significant changes in the stated markers of autonomic hemodynamic regulation.

\section{Methods}

\section{Subjects}

Fifty-five male participants (aged 18 - 22 years) enrolled for basic training at the South African National Defence Force (SANDF) were included in the study. Of the 55 males, 54 were black and 1 was of mixed race ethnicity. All participants had undergone mandatory physical examination and had been cleared to participate in intense physical activity. Exclusion criteria were a history of cardiovascular, hepatic, respiratory, renal, pulmonary, metabolic, or orthopaedic disease requiring medical attention, as well as any respiratory tract infection in the previous 2 weeks and use of any medication that could influence cardiovascular control. Although smoking was not an exclusion criteria, smoking history was recorded pre- and post-intervention, and none of the subjects reported a change in smoking habits. Physical characteristics of the participants are shown in Table 1. No professional athletes or highly trained individuals were included in this study. Baseline training status of participants varied from sedentary to infrequent recreational exercise training. After they were informed of the nature and aim of the study, all participants gave their written consent in accordance with ethics committee procedures at the University of Pretoria.

\section{Experimental protocol}

This was a prospective 20 -week exercise intervention study with a self-control design. The study protocol was submitted to and approved by the University of Pretoria Ethics Committee. The recording of cardiovascular variables was performed at week 1 (baseline) and again at week 20. No exercise was performed in the 24 hours preceding the testing and participants rested for a 10 -minute period before the testing. During the data sampling, participants were asked to remain as still and quiet as possible. This occurred in the absence of external auditory or visual stimuli. Room temperature was maintained at $20-22^{\circ} \mathrm{C}$. Sampling was done at $823 \mathrm{~m}$ above sea level. Blood pressure was then continuously sampled for 10 minutes in a supine position, of which the last 5 minutes of sampling were used for data analysis. All participants that started the study completed the intervention and were retested at week 20. The participants acted as their own control group as week 20 results were compared with baseline results.

\section{Exercise training programme}

The basic training programme conducted by the SANDF includes elements of aerobic and resistance training, as well as technical military skills training (Table 2). The primary aim of the programme was to develop basic fitness components such as cardiorespiratory and muscular endurance. Physical training includes upper and lower body strength, endurance and stretching exercise, as well as interval training and sustained marching or jogging for cardiovascular endurance. Technical skills training included drills, regimental aspects, compliments and saluting, musketry, shooting, signal training, mine awareness, map reading, buddy aid, field craft, water orientation, and parade rehearsal. The programme is progressive in intensity and duration, escalating at weeks $4,6,7,11$ and finally at week 13 . The calculated average basal metabolic rate (BMR) for participants, in addition to the energy expenditure of the training and exercise activities, resulted in a calculated average energy consumption of approximately $8485 \mathrm{~kJ} /$ day, which can be classified as a medium to high intensity exercise programme. ${ }^{[12]}$ Although other prospective exercise intervention studies vary from 6 weeks ${ }^{[13]}$ to 5 years, ${ }^{[11]}$ a 20 -week

Table 1. Physical characteristics and anthropometry of participants (week 1)

\begin{tabular}{ll}
\hline & Mean (SD) \\
\hline Age $(\mathrm{yr})$ & $20.9(1.2)$ \\
Height $(\mathrm{cm})$ & $171.0(7.1)$ \\
Mass $(\mathrm{kg})$ & $62.9(7.2)$ \\
BMI $\left(\mathrm{kg} / \mathrm{cm}^{2}\right)$ & $21.6(2.1)$ \\
Ethnicity & \\
$\quad$ Black & 54 \\
$\quad$ Mixed race & 1
\end{tabular}

exercise regimen was chosen for this study as this is the duration of the Basic Training Programme undertaken by the SANDF.

\section{Measurements}

The Finometer Pro (Finapres Medical Systems, the Netherlands) was used for data sampling. The Finometer PRO monitors blood pressure (BP) non-invasively with a volume clamp that is placed on the finger. When the volume clamp is activated the intra-arterial pressure equals that of the finger cuff pressure. Transmural pressure changes are observed and evaluated by a computer algorithm. The Finapres automatically tracks changes in the size of the arterial vessels caused by changes in the smooth muscle tone and makes available a calibrated pressure waveform which is interpreted by a pattern recognition

Table 2. PT programme

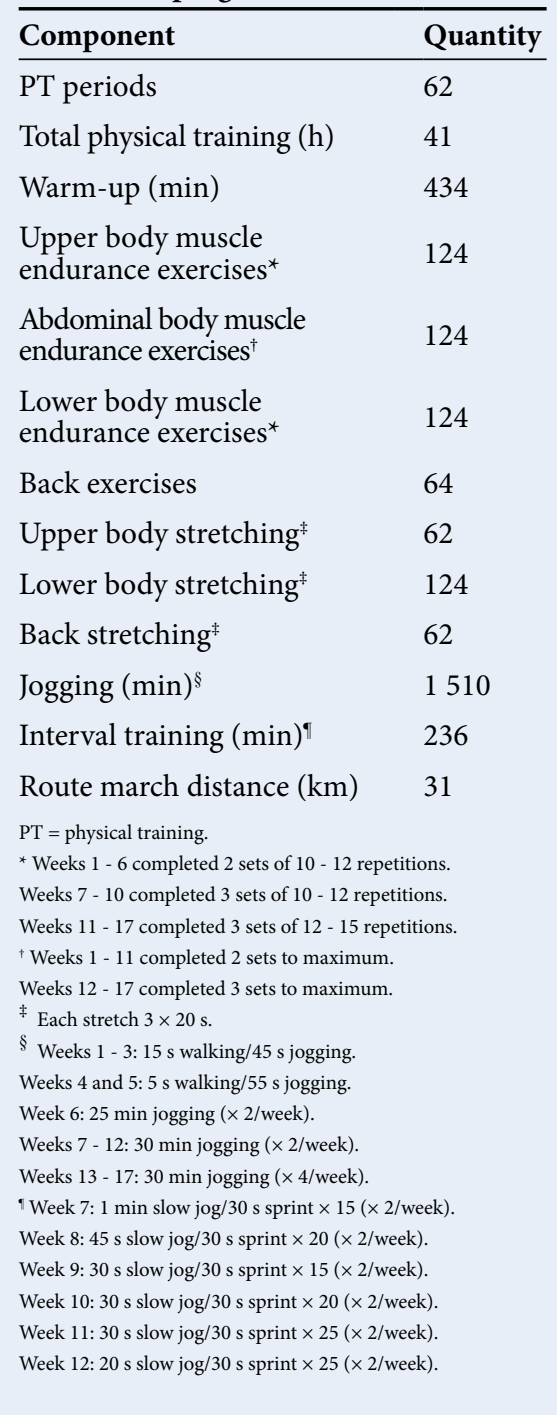


programme to provide SBP and DBP. ${ }^{[14]}$ Beatto-beat $\mathrm{BP}$ is then monitored over a chosen period. Additional model-flow calculations provided information on SV, CO and TPR. The 2010 Finapres BeatScope 1.1a software package (Finapres Medical Systems, the Netherlands) was used to quantify the following haemodynamic parameters: SBP, DBP, MAP and the variability (standard deviation, SD) of each, BP, CO, SV, HR, TPR, EJT, $\mathrm{ZaO}$ and Cwk. The Finometer Pro meets the Association for the Advancement of Medical Instruments accuracy requirements for automated sphygmomanometers, with the precision of results comparable with those published for auscultatory techniques. ${ }^{[15]}$ The Finometer is suitable for tracking blood pressure as within subject precision is high, 3.4 - $4.5 \mathrm{mmHG}$ for SBP, 1.5 - $2.0 \mathrm{mmHg}$ for $\mathrm{MBP}$ and 1.7 - $2.2 \mathrm{mmHg}$ for DBP at a $95 \%$ confidence interval. ${ }^{[16]}$

Measurement of exercise ability/fitness was determined by indirect calculation of $\mathrm{VO}_{2}$ max using the validated $2.4 \mathrm{~km}$ run test. ${ }^{[17]}$

\section{Statistical analysis}

As many of the variables follow a skew distribution, the results are reported using the median, interquartile range and the first and third quartiles. The Wilcoxon signed rank test, which is analogous to a paired sample $t$-test, was used to assess differences between baseline and week 20. A $p$-value $<0.05$ was considered statistically significant.

\section{Results}

The medians and quartiles of the cardiovascular and blood pressure variables evaluated are illustrated in Table 3 and Table 4 respectively.

\section{Heart rate, stroke volume and cardiac output}

The basic training programme resulted in a significant decrease in median resting $\mathrm{HR}$ by 9 bpm over 20 weeks $(p<0.01)$ (Table 3 ). Both SV and CO showed a significant response to exercise intervention with an increase of $22 \%(p<0.01)$ and $9 \%(p<0.05)$ respectively.

\section{Blood pressure and blood pressure variability}

Values of SBP, DBP, MAP and the SD are shown in Table 4 . These results show that after a 20 -week training programme, only

Table 3. Comparison of week 1 and week 20 cardiovascular variables measurements (supine)

\begin{tabular}{llll}
\hline Indicator & $\begin{array}{l}\text { Week 1, median } \\
(\boldsymbol{N = 5 5})\end{array}$ & $\begin{array}{l}\text { Week 20, median } \\
(\boldsymbol{N = 5 5})\end{array}$ & $\begin{array}{l}\text { p-value } \\
\text { Weeks 1 - 20 }\end{array}$ \\
\hline $\mathrm{VO}_{2} \mathrm{max}(\mathrm{mL} / \mathrm{kg} / \mathrm{min})$ & $55.68(53.74-58.09)$ & $58.15(57.16-60.39)$ & $<0.001^{\dagger}$ \\
$\mathrm{HR}(\mathrm{bpm})$ & $69.61(64.52-77.64)$ & $60.89(54.32-67.89)$ & $<0.001^{\dagger}$ \\
$\mathrm{SV}(\mathrm{mL} / \mathrm{beat})$ & $67.98(56.35-77.80)$ & $82.22(74.05-96.04)$ & $<0.001^{\dagger}$ \\
$\mathrm{CO}(\mathrm{Lpm})$ & $4.47(3.73-5.50)$ & $4.88(4.45-5.95)$ & $0.039^{\ddagger}$ \\
$\mathrm{EJT}(\mathrm{units})$ & $0.33(0.31-0.34)$ & $0.34(0.32-0.34)$ & $0.002^{\ddagger}$ \\
$\mathrm{TPR}(\mathrm{mmHg} / \mathrm{min} / \mathrm{L})$ & $1.05(0.83-1.37)$ & $0.94(0.76-1.04)$ & $0.003^{\ddagger}$ \\
$\mathrm{ZaO}(\mathrm{mMU})$ & $52.92(51.43-55.70)$ & $52.19(50.44-54.49)$ & $0.002^{\ddagger}$ \\
$\mathrm{Cwk}(\mathrm{MU})$ & $2.53(2.35-3.03)$ & $2.57(2.28-3.17)$ & 0.186 \\
$\mathrm{MU}=\mathrm{Medical} \mathrm{unit}(\mathrm{mmHH} . \mathrm{s} / \mathrm{ml})^{*}$ 25th -75 th percentile. & & \\
${ }^{+} p<0.001$. & &
\end{tabular}

Table 4. Comparison of week 1 and week 20 blood pressure indices measurements (supine)

\begin{tabular}{llll}
\hline Indicator $(\mathbf{m m H g})$ & $\begin{array}{l}\text { Week 1, median } \\
(\boldsymbol{N}=\mathbf{5 5})\end{array}$ & $\begin{array}{l}\text { Week 20, median } \\
(\boldsymbol{N = 5 5 )}\end{array}$ & $\begin{array}{l}\boldsymbol{p} \text {-value } \\
\text { Weeks 1 - 20 }\end{array}$ \\
\hline SBP & $101(91-111)$ & $103(97-110)$ & 0.186 \\
DBP & $65(60-71)$ & $60(57-68)$ & $<0.001^{\dagger}$ \\
MAP & $79(73-85)$ & $76(72-82)$ & 0.134 \\
MAP SD & $4.7(4.2-5.8)$ & $4.4(3.7-5.2)$ & 0.067 \\
SBP SD & $6.1(4.8-7.4)$ & $5.8(4.9-7.4)$ & 0.688 \\
DBP SD & $4.5(3.8-5.3)$ & $4.2(3.5-4.9)$ & 0.126 \\
${ }^{*} 25$ th - 75th percentile & & \\
${ }^{*} p<0.001$ (a priori statistical significance for all tests was set at $\left.\mathrm{p} \leq 0.05\right)$. & &
\end{tabular}

DBP decreased $(p<0.01)$. The changes in SBP and MAP were not statistically significant. BPV was assessed by using intra- and interparticipant $\mathrm{SD}$ and coefficient variation of arterial blood pressure. Although BPV did decrease in all measured variables, the change was not statistically significant.

\section{Ejection fraction, total peripheral} resistance, ascending aorta impedance and arterial compliance

As illustrated in Table 3, there were significant changes seen in EJT, TPR and $\mathrm{ZaO}(p<0.05)$ while Cwk remained unchanged.

\section{Aerobic capacity}

There was a significant $(p<0.001)$ improvement in $\mathrm{VO}_{2}$ max after the 20 -week training programme (Table 3 ). Although this study does not specifically focus on changes in $\mathrm{VO}_{2}$ max, it is important to document the training effect and consider all other results in the context of an improvement in aerobic capacity.

\section{Discussion}

A significant improvement in several measurements of cardiovascular function occurred after a 20-week aerobic and resistance training programme in this young and healthy group of participants.

Long-term aerobic activity is associated with haemodynamic and physiological adaptations of the cardiovascular system. ${ }^{[1,2,9,10]}$ The improvements in HR, SV, CO and EJT in this study indicate improved cardiac efficiency and are consistent with other studies conducted in young, healthy subjects. ${ }^{[9]}$ There are numerous studies documenting an increased SV after a prolonged exercise intervention in both older $^{[18]}$ and young, healthy subjects ${ }^{[9]}$ but specific detailed studies on black Africans are scant. Vella and Robergs ${ }^{[19]}$ suggest that SV response to exercise is influenced by training status, age and sex, but fail to discuss the influence of ethnicity. The present study provides valuable evidence that prolonged exercise induces improvements in SV in subjects of black African ethnicity. 
Improvements in aerobic fitness (measured by $\mathrm{VO}_{2} \mathrm{max}$ ) are associated with a decreased relative cardiovascular risk profile. ${ }^{[8]}$ In this group of healthy subjects, $\mathrm{VO}_{2}$ max increased by $4 \%$ between week 1 and week 20. Other studies conducted in young, healthy, non-African subjects have shown greater improvements in $\mathrm{VO}_{2}$ max following an exercise intervention, ${ }^{[20]}$ while a study conducted in young normotensive African-American men (aged 18 - 26 years) showed a $16 \%$ improvement in aerobic capacity following an 8 -week aerobic training programme. ${ }^{[1]}$ The main differences between this study and other studies was the baseline average body mass index (BMI) of subjects $\left(21.6 \mathrm{~kg} / \mathrm{cm}^{2}\right.$ compared with $25.0 \mathrm{~kg} / \mathrm{cm}^{2[1]}$ and $\left.25.3 \mathrm{~kg} / \mathrm{cm}^{2[20]}\right)$ and the type, duration and intensity of training performed (mixed resistance and aerobic training at medium to high intensity for 20 weeks v. aerobic training at high intensity for 6 days ${ }^{[20]}$ and 8 weeks ${ }^{[1]}$ ).

Regular exercise is associated with a beneficial vascular profile. ${ }^{[8]}$ The present study demonstrates that prolonged regular exercise causes beneficial changes in central $(\mathrm{ZaO})$ and peripheral (TPR) vasculature in young African males. Studies that have evaluated the effect of exercise on vasculature indeed show beneficial changes in both older ${ }^{[5,8]}$ and younger participants, ${ }^{[5]}$ with the effect of exercise on TPR and $\mathrm{ZaO}$ being seen as early as $4-6$ weeks in young, healthy participants. ${ }^{[13]}$ No change in Cwk was demonstrated in this study. This is consistent with a previous study conducted on young (22-yearold) African-American men, which showed no change in central artery stiffness after 6 weeks of training. ${ }^{[2]}$ Age has been identified as a determinant of arterial response to exercise, with younger subjects showing a greater response at peripheral vessel level, while older subjects experience predominant changes in large arteries. ${ }^{[5]}$ This may explain the results of the present study, which shows a change in arterial $\mathrm{ZaO}$, but not Cwk. The results suggest that $\mathrm{Cwk}$ is well maintained at a young age regardless of training status or aerobic fitness.

Epidemiological studies show there is a higher incidence of hypertension in black African populations compared with causasian populations and that exercise may play a beneficial role in reducing this incidence. ${ }^{[4]}$ We hypothesised that a 20 -week training programme would have improved blood pressure variables when compared with week 1, instead we found that SBP and MAP remained unchanged, and only the decrease in DBP was statistically significant (Table 4). Week 1 measurements of resting blood pressure showed that only one participant had an elevated SBP $(152 \mathrm{mmHg})$. By week 20 , the maximum SBP recorded was $131 \mathrm{mmHg}$. Other than this isolated elevated initial SBP, all participants were normotensive before the intervention. Most studies that investigate the relationship between exercise and cardiovascular efficiency have been conducted on older populations and many show significant decreases in both SBP and DBP. ${ }^{[21]}$ In the few studies that have been conducted among young healthy populations, these changes are less dramatic. As in the present study, differences may only be seen in $\mathrm{DBP},{ }^{[5]}$ and in some cases no change was seen across any of the blood pressure indicators, ${ }^{[13]}$ which may suggest that either changes in blood pressure may be dependent on age ${ }^{[5]}$ or that the margin for improvement in normotensive patients is narrower than in hypertensive patients.

$\mathrm{BPV}$ reflects vascular autonomic function and is a recognised marker of cardiovascular health. ${ }^{[22]}$ Studies have shown an elevated $\mathrm{BPV}$ to be an independent predictor of cardiovascular mortality. ${ }^{[22]}$ In this group of participants, BPV did not change significantly after 20 weeks of training. Our findings differ from a previous study on young normotensive males, which demonstrated significant improvement in autonomic blood pressure control systems following an 8-week exercise intervention. ${ }^{[1]}$ The difference in outcome between the Bond et al. ${ }^{[1]}$ study and the present study could be attributed to the pre-intervention physical activity level of the participants. The subjects in both studies were of similar mean (SD) ages (20 (2) years compared with 21 (1) years in the present study); however in the Bond et al. ${ }^{[1]}$ study,the participants were only included with the criterion that they did not participate in regular exercise during the previous year while our study included subjects with variable training history. It is also possible that improvements in autonomic modulation of blood pressure are best appreciated during physical or mental stress testing rather than at rest; some changes may have been seen if stress testing was included in this study protocol. In other studies that have shown a decreased BPV (and increased baroreceptor sensitivity) after prolonged training, participants were either all over the age of 40 or had comorbid illnesses. ${ }^{[23]}$ As such it is plausible that in a young, healthy population, good autonomic modulation is preserved and the margin for improvement with exercise will always be limited and seldom be significant. Conversely, in an elderly population or in a population with comorbidities affecting the cardiovascular system, such as diabetes or recent myocardial infarction, sustained exercise causes numerous beneficial changes to vasculature and neural modulation, which cumulatively result in a significant decrease in BPV post intervention. ${ }^{[22]}$ There are, however, a minority of studies that demonstrate no significant change in BPV following low intensity regular exercise in the elderly. ${ }^{[1]]}$ In these studies that showed no change in BPV, the participants tested had no comorbidities, corroborating the theory that a lack of comorbid illness could influence the amount of change expected from an exercise intervention.

\section{Study limitations}

This study was designed as a self-control study. It may have been strengthened by inclusion of a control group to verify that changes seen were solely due to the intervention. The study was conducted on army recruits who were subjected to a 20 -week training programme that increased in dose from week to week. Results are only reported for week 1 and week 20, giving no insight to what happened during this period. As such, one cannot differentiate the effects of duration of training from the dose of training. Finally, it may have been valuable to include physical or mental stress tests as a final means of validating any changes in markers of autonomic blood pressure control such as BPV.

\section{Conclusion}

This study contributes to the growing pool of research conducted among healthy, young individuals providing insights to how ethnicity may or may not influence findings. The findings in this study suggest that exercise improves the vascular profile among African men even at a young age. Changes in CO, SV, TPS, EJT and Cwk suggest a favourable response to exercise and a reduced risk for cardiovascular disease. The fact that SBP and the variability indicators (SBP SD, DBP SD) remained unchanged is most likely related to the age, general health status and pre-intervention training status of the participants. Results suggest that young age and lack of comorbidity decreases variance in BPV after a training intervention. Further cross-sectional studies among trained 
and untrained youths need to be conducted to corroborate this theory. Previous research suggests that even small changes in cardiovascular indices sustained over a prolonged period will be beneficial to overall and long-term wellbeing. The findings in this study, in conjunction with all research cited in this article, can be used as evidence to support life-long aerobic exercise as a possible non-pharmacological cardioprotective therapy in subjects of all ethnic groups.

Acknowledgements. Lt Col E Terblanche, Wing Commander: Research and Development Joint Physical Training, Sport and Recreation Training Centre, South African National Defence Force.

\section{References}

1. Bond V, Bartels MN, Sloan RP, et al. Exercise training favourably affects autonomic and blood pressure responses during mental and physical stressors in AfricanAmerican men. J Hum Hypertens 2009;23(4):267-273. [http://dx.doi.org/10.1038/ jhh.2008.125]

2. Heffernan KS, Fahs CA, Iwamoto GA, et al. Resistance exercise training reduces central blood pressure and improves microvascular function in African American and white men. Atherosclerosis 2009;207(1):220-226. [http://dx.doi.org/10.1016/j. atherosclerosis.2009.03.043]

3. Swift DL, Johannsen NM, Lavie CJ, et al. Racial differences in the response of cardiorespiratory fitness to aerobic exercise training in Caucasian and African American postmenopausal women. J Appl Physiol 2013;114(10):1375-1382. [http:// dx.doi.org/10.1152/japplphysiol.01077.2012]

4. Heffernan KS, Jae SY, Vieira VJ, et al. C-reactive protein and cardiac vagal activity following resistance exercise training in young African-American and white men. Am J Physiol Regul Integr Comp Physiol 2009;296(4):R1098-R1105. [http://dx.doi. org/10.1152/ajpregu.90936.2008]

5. McDonnell BJ, Maki-Petaja KM, Munnery M, et al. Habitual exercise and blood pressure: Age dependancy and underlying mechanisms. Am J Hypertens 2013;26(3):334-341. [http://dx.doi.org/10.1093/ajh/hps055]

6. Assah FK, Ekelund U, Brage S, Mbanya JC, Wareham NJ. Urbanization, physical activity, and metabolic health in sub-Saharan Africa. Diabetes Care 2011;34(2):491496. [http://dx.doi.org/10.2337/dc10-0990]

7. Price JH, Khubchandani J, McKinney M, Braun R. Racial/ethnic disparities in chronic diseases of youths and access to health care in the United States. Biomed Res Int 2013;Article ID 787616:12 pages. [http://dx.doi.org/10.1155/2013/787616]

8. Thijssen DH, Maiorana AJ, O’Driscoll G, Cable NT, Hopman MT, Green DJ. Impact of inactivity and exercise on the vasculature in humans. Eur J Appl Physiol 2010;108(5):845-875. [http://dx.doi.org/10.1007/s00421-009-1260-x]
9. Heydari M, Boutcher YN, Boutcher SH. High-intensity intermittent exercise and cardiovascular and autonomic function. Clin Auton Res 2013;23(1):57-65. [http:// dx.doi.org/10.1007/s10286-012-0179-1]

10. Prista A, Macucule CF, Queiroz AC, et al. A bout of resistance exercise following the 2007 AHA guidelines decreases asleep blood pressure in Mozambican men. J Strength Cond Res 2013;27(3):786-792. [http://dx.doi.org/10.1519/JSC.0b013e31825d9783]

11. Uusitalo ALT, Laitinen T, Vaisanen SB, Lansimies E, Rauramaa R. Physical training and heart rate and blood pressure variability: A 5-yr randomized trial. Am J Physiol-Heart Circ Physiol 2004;286(5):H1821-H1826. [http://dx.doi.org/10.1152/ajpheart.00600.2003]

12. Foss ML, Keteyian SJ. Fox's Physiological Basis for Exercise and Sport. 6th ed. Iowa: McGraw-Hill, 1998.

13. Parshad O, Richards A, Asnani M. Impact of yoga on haemodynamic function in healthy medical students. W Indian Med J 2011;60(2):148-152.

14. Langewouters GJ, Settels JJ, Roelandt R, Wesseling KH. Why use Finapres or Portapres rather than intraarterial or intermittent non-invasive techniques of blood pressure measurement? J Med Eng Technol 1998;22(1):37-43. [http://dx.doi.org/10.3109/03091909809009997]

15. Guelen I, Westerhof BE, van der Sar GL, et al. Finometer, finger pressure measurements with the possibility to reconstruct brachial pressure. Blood Press Monit 2002;8(1):2730. [http://dx.doi.org/10.1097/00126097-200302000-00006]

16. Imholz BP, Weiling W, Langewouters GJ, Montfrans GA. Continuous finger arterial pressure: Utility in the cardiovascular laboratory. Clin Auton Res 1991;1(1):43-45. [http://dx.doi.org/10.1007/BF01826057]

17. Burger SC, Bertram SR, Stewart RI. Assessment of the $2.4 \mathrm{~km}$ run as a predictor of aerobic capacity. S Afr Med J 1990;78(6):327-329.

18. Cornelissen VA, Goetschalckx K, Verheyden B, et al. Effects of endurance training on blood pressure regulation, biomarkers and the heart in subjects at a higher age. Scand J Med Sci Spor 2011;21(4):526-534. [http://dx.doi.org/10.1111/j.16000838.2010.01094.x]

19. Vella CA, Robergs RA. A review of stroke volume response to upright exercise in healthy subjects. Br J Sport Med 2005;39(4):190-196. [http://dx.doi.org/10.1136/ bjsm.2004.013037]

20. Astorino TA, Allen RP, Roberson DW, Jurancich M. Effect of high-intensity interval training on cardiovascular function, $\mathrm{VO}_{2}$ max, and muscular force. J Strength Cond Res 2012;26(1):138-145. [http://dx.doi.org/10.1519/JSC.0b013e318218dd77]

21. Davis WJ, Wood DT, Andrews RG, Elkind LM, Davis WB. Concurrent training enhances athletes' strength, muscle endurance, and other measures. J Strength Cond Res 2008;22(5):1487-1502. [http://dx.doi.org/10.1519/JSC.0b013e3181739f08]

22. Galetta F, Franzoni F, Platinga Y, et al. Ambulatory blood pressure monitoring and endothelium-dependent vasodilation in the elderly patients. Biomed Pharmacother 2006;60(8):443-447. [http://dx.doi.org/10.1016/j.biopha.2006.07.013]

23. Spierer DK, DeMeersman RE, Kleinveld J, et al. Exercise training improves cardiovascular and autonomic profiles in HIV. Clin J Auton Res 2007;17(6):341-348. [http://dx.doi.org/10.1007/s10286-007-0441-0] 